\title{
Application of quantum master equation for long-term prognosis of asset-prices
}

\author{
Polina Khrennikova*, \\ University of Leicester, University Road, LE1 7RH.
}

November 20, 2015

\begin{abstract}
This study combines the disciplines of behavioral finance and an extension of econophysics, namely the concepts and mathematical structure of quantum physics. We apply the formalism of quantum theory to model the dynamics of some correlated financial assets, where the proposed model can be potentially applied for developing a long-term prognosis of asset price formation. At the informational level, the asset price states interact with each other by the means of a "financial bath". The latter is composed of agents' expectations about the future developments of asset prices on the finance market, as well as financially important information from mass-media, society, and politicians. One of the essential behavioral factors leading to the quantum-like dynamics of asset prices is the irrationality of agents' expectations operating on the finance market. These expectations lead to a deeper type of uncertainty concerning the future price dynamics of the assets, than given by a classical probability theory, e.g., in the framework of the classical financial mathematics, which is based on the theory of stochastic processes. The quantum dimension of the uncertainty in price dynamics is expressed in the form of the price-states superposition and entanglement between the prices of the different financial assets. In our model, the resolution of this deep quantum uncertainty is mathematically captured with the aid of the quantum master equation (its quantum Markov approximation). We illustrate our model of preparation of a future asset price prognosis by a numerical simulation, involving two correlated assets. Their returns interact more intensively, than understood by a classical statistical
\end{abstract}

*Email:pk198@le.ac.uk 
correlation. The model predictions can be extended to more complex models to obtain price configuration for multiple assets and portfolios.

Keywords: behavioral finance, decision making, quantum information and probability, violation of Bayesian rationality, open quantum systems.

\section{Introduction}

This paper is a contribution to the field of behavioral finance, with an emphasis on mathematical modeling of irrationality ${ }^{1}$ The problem that we search to approach in this study cannot be formulated better than in the following extended citation from Takahashi and Terano, [1]:

"Most conventional financial theories build the models by assuming representative rational investors based on the hypothesis that "even if each investor has different future prospects, these errors are cancelled out as a whole and do not affect the prices". However, the participants of real markets only have limited information and they do not necessarily process the obtained information appropriately. There is a good possibility that the market participants have a common bias that deviates from rationality. If this is the case, the deviation from rationality will not be cancelled out and will have a large impact on the prices. To unravel the mechanism of price fluctuation in real markets, it is quite important to understand the difference between the decision-making in the real world and the one based on the maximization of expected utility and to clarify how the prices are affected by the deviation from rationality on decision-making."

We proceed with modeling the formation of such biases ${ }^{2}$ with the aid of the novel approach based on exploring the formalism of quantum theory (and

\footnotetext{
${ }^{1}$ The concept of irrationality can be coupled to the notion "bounded rationality" coined by Herbert Simon ([4], [5] of finance market players. The deviations from the rational mode of processing the financial information and the implications it has for the formation of asset prices, will be discussed in section 5 .

${ }^{2}$ See also Shiller [8] who emphasized that financial markets are sometimes irrational as investors often make their decisions based on personal preferences and e.g. over-confidence bias. Irrationality of investors is actively explored in the studies related to the emergence of financial bubbles, e.g., Bailey [10], Kindleberger [6], [7]. It is beyond the scope of this work to concentrate on the specific interrelation, irrationality-bubbles. We proceed under the assumption that the investors may behave irrationally even in the absence of bubble-like events, see agent based model simulation by[2]. Of course, we understand well the role of financial bubbles and the subsequent downturns in increasing the interest to behavioral finance. Bubbles call for a behavioral explanation. The recent financial crisis of 2008 clearly demonstrated that strict opponents of the Efficient Market Hypothesis, claiming that bubbles are impossible in well-organized markets, where all financial information is publicly available, have neglected some factors behind the real price formation of financial assets. Behavioral factors seem to be determining for the price evolution even
} 
its methodology) outside of physics. Recently, this formalism was actively applied to a wide range of social science problems, especially in cognitive psychology, behavioral finance and decision making, see [29]-[43]. At heart of this formalism is the quantum probability theory, based on the calculus of complex probability amplitudes (or more generally density matrices). This calculus leads to a nonclassical scheme of probability update, different from the classical Bayesian scheme. Thus, the quantum approach is able to capture violations of Bayesian rationality. For example, in models of behavioral economics it is important to explain and model contexts for which the Savage Sure Thing Principle can be violated. Effectively, in a situation where one cannot presume that agents are "Baeysian rational", a deeper type of uncertainty than the uncertainty of the rational agents, reasoning and acting in accord with some probabilistic information, can be considered. In the mathematical formalism of quantum theory such nonclassical uncertainty is represented in the form of superposition of alternatives. Please, see remark 1 for an more extensive interpretation of the distinction, made between the classical and the quantum approaches to uncertainty.

Along with the other interdisciplinary applications, in this work, quantum physics is used as an operational formalism, describing statistical data generated by experiments. Its crucial difference from classical statistical theories is that it ignores all the parameters of the model, besides those directly related to the measurements. ${ }^{3}$

With this in mind, the superposition of state vectors, representing some choice alternatives, is just a mathematical tool to express uncertainty, which is deeper than the uncertainty described by a classical probability. The irrationality of decision making of the market agents that is manifest in the quantum superposition of their beliefs and decisions, is one of the main factors behind the deep quantum-like uncertainty in the formation of asset prices. Such a price formation of an asset is expressed in the form of superposition of the different prices and can yield a price that deviates from the fundamental value predicted by the classical finance theories. Another profoundly quan-

on informationally-efficient markets, see, e.g., Bailey [10].

${ }^{3}$ In quantum physics, one even employs the so called no-go theorems for such parameters, for example, see [11]. However, for our purposes, these theorems are not of such a value as in physics. Consider, for example, one of the most famous no-go theorems, on Bell's inequality [3], [11]. It states that if the "hidden parameters" were existing, they would be nonlocal. However, it seems that, e.g., for the financial markets, the issue of nonlocality is not critical. Definitely, financial correlations are nonlocal, but they are not super-luminary. Another no-go theorem is the Kochen-Specker theorem [11]. It says that if "hidden parameters" were existing, they would be contextual. Indeed, human behavior is intrinsically contextual and contextuality is a key feature of the quantum formalism, which is resonating with its notion in behavioral studies, cf. with [36] and [37]. 
tum information tool, which we use in our modeling is entanglement, i.e., the states' non-separability. The prices of a group of assets ( e.g. in a portfolio) can be correlated with each other and with a "financial bath", by correlations that are stronger than the possible classical probabilistic correlations.

We devise a model that dynamically captures the process of asset price formation and can be applied for preparing a long-term financial prognosis. A decision maker can be e.g., an individual financial consultant, trader, financial forecast agency, or a financial trading corporation. In order to develop some future trading strategy the decision maker (we refer to her as a financial expert) would need to come with a prognosis for a group of asset prices. This model can be expanded for forecasting the movements of different types of financial instruments, e.g., currency exchange rates). We model the process of decision making related to the asset price formation. The resolution of uncertainty in future prices of assets is described with the aid of the theory of open quantum systems [50]: the most powerful and general apparatus to describe adaptive dynamics of a system (in our case assets) interacting with some bath (in this representation, the bath is of a purely informational nature, consisting of the expectations of the agents acting on the finance market, the mass-media news and other signals of financial, social, and political origins that can be regarded as "events" to contribute to price change).

The original dynamical equation from the theory of open quantum systems, the quantum master equation, is too complex, and typically physicists use its (quantum) Markov approximation [50]. We also work with this quantum Markovian dynamics. In physics the process of the resolution of uncertainty of the superposition-entanglement type leading to the equilibrium state is called decoherence. We emphasize that the process of decoherence of the asset price states into their equilibrium price state is essential in the presented model. If a devised model would not involve decoherence then a superposition of various asset prices would fluctuate and the decision maker would not be able to make the concrete investment decision. We can draw a comparison with quantum physics to elucidate the importance of decoherence in this setting. Decoherence is one of natural scenarios of the determination of the concrete value of a quantum observable, in the so called decoherence model of measurement, [44]. Another approach to quantum measurement is based on the notion of state reduction (collapse). In cognitive modelling the latter approach, i.e., instantaneous decision making, does not correspond well to the real nature of cognitive processes. The main feature of the cognitive processes, including decision making, is that they have a finite time duration until a concrete decision is reached.

In the setting of this work the reader will find a financial application of 
a general theory of decision as decoherence developed in a series of papers of Asano et al., e.g., [29], [30], who modeled, e.g., irrationality of behavior of players in games of the Prisoners Dilemma type. This approach, based on theory of open quantum systems, is a part of the generalized quantum theory models of decision making [32].

During the recent years, quantum probability and decision making were successfully applied to describe a variety of problems, paradoxes, and probability judgment fallacies, e.g., conjunction and disjunction effects, Allais paradox ( violations of Von Neumann-Morgenstern expected utility axioms), Ellsberg paradox ( violation of Aumann-Savage subjective utility axioms), see, e.g., [29]-[43].

In the standard Capital Asset Pricing Model (CAPM) the equilibrium asset price is derived on the assumptions of rational investors [45], [9] and the mean variance efficiency of the market. However, this model as well the traditional financial theories ( among them various extensions of CAPM, with additional explanatory loading factors, [46], [47]) have been criticized in terms of their explanatory power and the validity of their assumptions [1]: Even when CAPM was suggested, Simon already pointed that the rationality of human being is bounded [4]. However, an asset price in financial markets is considered to be priced based on the fundamental value for the reasons that "irrational investors behave random and their behaviors are cancelled out" and "arbitrage transactions adjust the market price to the fundamental value".

At the same time, [1] advocate that the assumptions of traditional financial theories have been questioned by the behavioral finance school, by pointing that "there are good reasons to believe that the behavior of irrational investors is biased" and "the capability of arbitrage is limited". Our approach based on the quantum adaptive dynamics, representing adaptivity of prices to beliefs and expectations of investors can be considered as an extension of CAPM giving a possibility to include irrationality and biases into the time dynamics of the asset prices, approaching the state of an equilibrium ${ }^{4}$. The ideology of our model is similar to the notion of "noise traders", applied as a point of reference in the so called "Behavioral CAPM", [48]. We emphasize that we search to depict the dynamics of the asset prices, not as reflections of some objective factors ( e.g. as $\mathrm{P} / \mathrm{B}$ ratio or firm size), but as the result of the beliefs of investors about the value of the asset that can deviate from its fundamental value. The traders can be categorized as bounded rational, as a result of their non-Bayesian mode of processing the financial informa-

\footnotetext{
${ }^{4}$ This final price realization state is not changed by further inputs from the financial environment.
} 
tion, as well as other factors, such as reluctance to perform such an analysis and instead the usage of heuristics, by coping the behavior of other traders (aka herding bias). Further, the investors are not perceiving the systematic risk of the market in a rational way and thus act over-confident and overoptimistic, or vice versa. The complexity of information and a lack of transparency can't be neglected either. It has been recognized that the financial markets can periodically ( or constantly, depending on the type of market) deviate from all forms of Market Efficiency, in particular the semi-strong and the strong forms, see a comprehensive account in [49]. All the aforementioned factors contribute to the formation of a special system of beliefs of the investors that shape their investment patterns. In this work we go beyond the classical Markovian models of investors probabilistic beliefs' evolution and appeal to the quantum formalism, to capture the non-classical processing of information. The beliefs of the investors are in a sense creating the price of the asset that often deviates from the mean - variance efficiency standards of classical financial models. We illustrate our model of preparation of a prognosis (by taking into account the "financial bath") by a numerical simulation. This is an illustrative example for the prognosis of the dynamics of prices of two correlated assets that is adaptive, with respect to the financial bath. Here we explore a mathematical construction borrowed from quantum physics: the Markov dynamics of systems of ions, interacting with the radiation background.

In general, by applying the mathematical apparatus of quantum theory outside of physics brings one to a very advantageous position, in terms of being able to utilize the advanced tools and models developed in statistical and quantum physics over the last hundred of years, by endowing them with novel interpretations; in our case the financial one. This is a general idea of econophysics, [14], where, for example, the methods of classical statistical physics were successfully explored in economics and finance over the last decades. This paper can be considered as a contribution to the rapidly expanding area of econophysics, quantum finance and quantum economics see, e.g., [15]-[27].

In line with these contributions to finance, we search to unify the methods of (quantum) econophysics with dynamical decision making and behavioral finance.

Finally, we remark that the proposed formalism is based on a financial analogue of a Fock space, where assets' prices play the role of numbers of quantum particles with specific states. Such a space is infinite dimensional, i.e., in the theoretical model prices can grow up to infinity. The application of the infinite dimensional space is merely a computational tool this allowing for a mathematical convenience. It is always possible to consider 
finite dimensional subspaces of the financial Fock space as, e.g., we do in our numerical simulation, in section 4 . The appropriate state space can be derived empirically, by analyzing the historical price statistics of the particular assets.

\section{Quantum representation of financial asset- prices}

In this representation, we proceed with a discrete price model. It is assumed that there is chosen some monetary unit, say dollar, and all prices are expressed in terms of this unit, i.e., they are given by natural numbers, $n=1,2, \ldots$ If the price of a financial asset is less than one dollar, then it is identified with the discrete level $n=0$, i.e., in this model the price level $n=0$ does not correspond to complete collapse of a financial asset in terms of its value. We emphasize that the model allows for a long term prognosis ( at some future point in time) of the dynamics of $N$ financial assets $\mathcal{A}_{j}: j=1,2, \ldots, N$.

\subsection{State space of single asset}

In the real finance market, at each moment of time, each single asset $\mathcal{A}_{j}$ has the definite price $p_{j}$. This quantity can be treated as classical (as any result of measurement in quantum theory). However, in the process of a prognosis of asset's price this assumption, about the fixed ("objective") price of an asset, is too constrained. Even when one considers classical finance theories, an asset's price is modeled probabilistically, e.g., with the aid of theory of stochastic processes [13]. The quantum approach gives a possibility to model a deeper type of uncertainty, associated with the asset price determination. Such type of an intrinsic uncertainty of the actual price is represented by superposition of the fixed-price states. ${ }^{5}$

The state space of a single financial asset $\mathcal{A}_{j}$ is infinite dimensional Hilbert space $H_{j}$ with the basis $\left|n_{j}\right\rangle, n_{j}=0,1,2, \ldots$. As was pointed out, besides the fixed price states given by the basis vectors $\left|n_{j}\right\rangle$, this space contains

\footnotetext{
${ }^{5}$ It important to remark that the classical probabilistic uncertainty of the price values can be in a similar mode modeled with the aid of the quantum formalism - by using so-called mixed states, statistical mixtures of fixed price states. However, for a moment we prefer to proceed with pure states (although the model that is applied poses a need to introduce mixed states as well, since the decision making dynamics driven by the quantum master equation transfers a pure state into a mixed state).
} 
superpositions of these states of the form:

$$
|\psi\rangle=\sum_{n_{j}} c_{n_{j}}\left|n_{j}\right\rangle
$$

where $c_{n_{j}}$ are complex numbers, $\sum_{n_{j}}\left|c_{n_{j}}\right|^{2}=1$. Here $\left|c_{n_{j}}\right|^{2}$ give the probabilities $P_{n_{j}}$ that the $j$ th asset has the price $n_{j}$.

In such a quantum structure the complex numbers have not only amplitudes, but also phases, $c_{n_{j}}=\left|c_{n_{j}}\right| e^{i \theta_{n_{j}}}$. In the quantum formalism the phases, more precisely the relative phase $\theta_{n_{j_{1}}}-\theta_{n_{j_{2}}}$, play an important role. The presence of relative phases contribute nontrivially to the state dynamics, that can be either Schrödinger dynamics or dynamics based on the quantum master equation. The latter is taking into account interaction with a "market expectations environment" representing the expectations of agents acting at the market, see sections 3.2, 3.3 for further consideration. In this sense such a quantum dynamics differs crucially from a classical Markovian state dynamics, which takes into account only the probabilities $P_{n_{j}}$.

\subsection{State space for multi-asset price configuration}

As in standard QM representation, the complete price configuration of $N$ assets is depicted as the tensor product of the state spaces $H_{j}$ :

$$
H=\otimes_{j=1}^{N} H_{j} .
$$

Its vectors have the form:

$$
|\Psi\rangle=\sum_{n} C_{n}|n\rangle
$$

where $n=n_{1} \ldots n_{N}$ and $\sum_{n}\left|C_{n}\right|^{2}=1$.

This state space represents another purely quantum information effect (which is not reduced to states superposition), namely, entanglement: entanglement of the asset-prices for different assets.

The states of the space $H$ can be separable and non-separable (entangled). Separable states that can be split into isolated states, each in its respective state space, can be represented as:

$$
|\psi\rangle=\otimes_{j=1}^{N}\left|\psi_{j}\right\rangle=\left|\psi_{1} \ldots \psi_{N}\right\rangle,
$$

where $\left|\psi_{j}\right\rangle \in H_{j}$. (For a moment, we consider only pure states.) The states which cannot be represented in this way are called non-separable, entangled. 
The degree of entanglement can be quantified with the aid of various measures. Their precise mathematical form is not important for our considerations, see, e.g., [11] for details. We just point to the existence of a possibility to order these states with the aid of some fixed measure of entanglement. In particular, we can speak about maximally entangled states. For example, consider two assets $\mathcal{A}_{1}$ and $\mathcal{A}_{2}$ and two prices $k_{1}$ and $k_{2}$ for $\mathcal{A}_{1}$ and $m_{1}$ and $m_{2}$ for $\mathcal{A}_{2}$. Then so-called Bell's states

$$
\begin{aligned}
& \left|\Phi^{+}\right\rangle=\left(\left|k_{1} m_{1}\right\rangle+\left|k_{2} m_{2}\right\rangle\right) / \sqrt{2} ; \quad\left|\Phi^{-}\right\rangle=\left(\left|k_{1} m_{1}\right\rangle-\left|k_{2} m_{2}\right\rangle\right) / \sqrt{2} \\
& \left|\Psi^{+}\right\rangle=\left(\left|k_{1} m_{2}\right\rangle+\left|k_{2} m_{1}\right\rangle\right) / \sqrt{2} ; \quad\left|\Psi^{-}\right\rangle=\left(\left|k_{1} m_{1}\right\rangle+\left|k_{2} m_{2}\right\rangle\right) / \sqrt{2}
\end{aligned}
$$

are entangled. In the case of the infinite dimensional asset-price state space the price states of assets are not maximally entangled. However, if there were only two possible price configurations for each asset, i.e., $k_{1}, k_{2}$ and $m_{1}, m_{2}$, respectively, then such states can be maximally entangled, giving rise to Bell states.

From the interpretational viewpoint, the notion of entanglement is one of the most complicated and debated themes in quantum physics. One of the features of entanglement (in the framework of our model) is that generally, in the process of preparing a forecast of asset-prices, it is impossible to treat the asset price dynamics isolation. Moreover, it is even impossible to treat the prices and returns of assets as classically correlated, as obtained through the covariance - variance relation. The correlations, which are encoded in an entangled state, are stronger than correlations described by the classical probabilistic models, see, e.g., [11].

Finally, we remark that even separable asset price state carries an essential degree of quantumness (the associated uncertainty that we discuss in Remark 1) related to the effect of superposition.

Remark 1: Classical and quantum representation of uncertainty Classical uncertainty is mathematically modeled with the aid of probability measures. Quantum uncertainty is mathematically modelled with aid of state superposition. What is the main interpretational difference between these two approaches, especially in applications of quantum-like models to finances? Classical probability measure accounts chances of realization of actual states of the financial market. For example, consider two assets $\mathcal{A}_{\infty}$ and $\mathcal{A}_{\in}$ with possible prices $n_{1}=0,1,2, \ldots$ and $n_{2}=0,1,2 \ldots$ We can consider some classical probability measure $p$ defined on the configurations space of possible actual states of the market, $\Omega=\left\{\omega=\left(n_{1} n_{2}\right)\right\}$. Here $p(\omega)$ quantifies the chance of realization of the actual price configuration $\omega$. Consider now some quantum superposition, e.g., $c_{1}\left|k_{1} m_{1}\right\rangle+c_{2}\left|k_{2} m_{2}\right\rangle$. This states encodes 
expert's beliefs in chances of realization of the actual states of the financial market. In her belief state space both price configurations $\left(k_{1}, m_{1}\right)$ and $\left(k_{2}, m_{2}\right)$ can coexist peacefully . A the same the classical probability measure $p$ encodes the "either-or" situation. This difference between classical and quantum uncertainties motivates for the use of the terminology "deeper uncertainty" for quantum uncertainty, as an uncertainty about uncertainty.

\section{Decision making: asset-prices prognosis}

An expert, decision maker, that searches to come with a long term prognosis of prices for multi-asset configurations, i.e., with probabilities that the price configuration of assets will be characterized by the vector of prices $|n\rangle=\left|n_{1} \ldots n_{N}\right\rangle$. As such her aim is to resolve the uncertainty encoded in a superposition (2). In financial terminology, the expert can analyze the output of the model, which provides the expert with the following information: the time of asset price stabilization and the associated probabilities of the possible future prices. Naturally, this data enables the expert to establish the future price configuration of an asset or a series of assets at a given time point. Technically, the time of asset price fluctuations in the proposed model is going to infinity. The fluctuations of the asset prices are damped under the pressure of "expectations bath", but the small price fluctuations still persist. This property of the model demands the expert to fix the exact timing of the equilibrium of state prices fluctuations in an artificial manner, by setting some fluctuation threshold. We discuss the model structure in more detail in the very end of section 3 .

In the quantum formalism the dynamics of the state of an isolated quantum system is described by the Schrödinger's equation and the dynamics of the state of a quantum system interacting with an environment is described by the quantum master equation [50]. In general the latter equation is too complicated mathematically to treat it straightforwardly i.e., without using some approximations. Therefore, various approximations are used. The most popular is the quantum Markovian approximation leading to the Gorini-Kossakowski-Sudarshan-Lindblad (GKSL) equation [50]. We will discuss its features and outline the mathematical structure in section, 3.3 .

\subsection{Expectations as a bath}

By applying the mathematical formalism of quantum physics to the problem of the asset-price prognosis one has to specify the notions of "isolation" and "environment". We start with the notion of environment. We point out that 
our approach to asset pricing is concerned with the information dynamics, the information about the possible asset-prices in a future point in time. For this specific purpose, it is natural to identify the environment, with the "bath" of expectations of the agents on the finance market. The notion of "isolated dynamics" corresponds to the ignorance of the impact of such a bath and the assets' price interaction. Effectively, in physics the notion of an isolated quantum system is merely an abstraction. In reality no completely isolated systems would exist. If we consider quantum field theory, even the vacuum has to be treated as an environment generating an influence on the photons. However, in some contexts one can ignore the impact of the environment with some degree of approximation. By considering the sources of a system's dynamics, we can split the generator of dynamics into two parts; the first one corresponding to the ignorance of the environment ( this would generate a Schrödinger's dynamics) and the second generator is encoding the impact of the "market expectations environment".

In the model under construction, a decision maker working on the long term prognosis of the asset price dynamics of a group of financial assets, analyzes the expectations of the finance market participants. Since the expectations of the agents often create market prices that don't correspond to the real values of the assets (and even if these values coincide at a given point in time, the real asset prices, as understood by the classical finance theory, are not sustainable over time) this model can be considered as a part of the field of behavioral finance and economics. The analysis of investors' expectations about the market prices, rather than the analysis of the company's fundamentals, plays a key role in the generation of the probabilistic prognosis of the future prices in this setting.

\subsection{Prognosis of the asset price dynamics without the expectations of investors}

In the process of asset pricing, the decision maker surely analyzes the price dynamics of each single financial asset as well as their mutual interactions. However, typically such an analysis is not sufficient to come to the concrete financial prognosis, since the internal characteristics of the assets don't encode all the factors impacting upon its market price. This situation can be mathematically expressed as fluctuations of the solutions of Schrödinger's equation:

$$
\frac{\gamma}{i} \frac{d \Psi}{d t}(t)=\mathcal{H} \Psi(t)
$$

where $\mathcal{H}$ is a Hermitian operator acting in $H$ and generating the state dynamics, without accounting for the impact of an environment and $\gamma>0$ is 
a factor determining the time scale of the dynamics (if the $\mathcal{H}$ is chosen as a dimensionless quantity).

Remark 1. (On the meaning of the scaling factor $\gamma$ ) In quantum mechanics the Hamiltonian, $\mathcal{H}$ has the dimension of energy and $\gamma=\hbar$ is the reduced Planck constant $\hbar=h / 2 \pi$. It has the dimension of action $=$ energy $\times$ time. One may attempt to proceed in the same way by inventing the notion of the "energy of market expectations" which would be a quite natural approach. However, the main problem of this approach is the development of the measurement methodology for such a kind of the "mental energy." This is a complicated problem related to the interdisciplinary application of physical models. We postpone a more broad discussion to future publications. Nevertheless, heuristically it is convenient to refer to the "energy of agents' expectations" to couple the abstract quantum formalism with the process of investment decision making in finance.

In the absence of "interactions" between assets prices the Schrödinger's dynamics is described by the Hamiltonian of the form

$$
\mathcal{H}_{0}=\mathcal{H}_{01} \otimes I+I \otimes \mathcal{H}_{02}
$$

where $\mathcal{H}_{0 j}: H_{j} \rightarrow H_{j}, j=1,2$, are Hamiltonians generating the price dynamics of different assets. These free Hamiltonians are typically defined with the aid of "number operators"

$$
N_{j}\left|n_{i}\right\rangle=n_{i}\left|n_{i}\right\rangle, n_{i}=1,2, \ldots
$$

which are diagonal in the basis $\left(\left|n_{i}\right\rangle\right)$. Then

$$
\mathcal{H}_{0 j}=E_{j} N_{j}
$$

where the parameters $E_{j}$ plays the role of the discussed above "energy of expectations" of the agents on the financial market for the price $p_{j}=n_{j}$ of the financial asset $\mathcal{A}_{j}$ In the state space of $j$ th asset, such free Hamiltonian generates the dynamics of the form

$$
\psi(t)=\sum_{n_{j}} c_{0 n_{j}} e^{-i t E_{j} n_{j} / \gamma}\left|n_{j}\right\rangle
$$

where $\Psi_{0}=\sum_{n_{j}} c_{0 n_{j}}\left|n_{j}\right\rangle, \sum_{n_{j}}\left|c_{0 n_{j}}\right|^{2}=1$ is the initial state. From this representation we can see that the solutions of Schrödinger's equation different from the stationary ones will fluctuate forever, i.e. no stabilization to the definite state will take place. This feature of Schrödinger's dynamics doesn't 
allow for its application in the process of preparation of a financial prognosis. Moreover, such a fluctuating dynamics cannot resolve the deep quantum ("intrinsic") uncertainty encoded in superposition and entanglement of the price states.

In the price basis the operator $\mathcal{H}_{0}$ is diagonal. Interaction between assetprices is described by non-diagonal operators coupling the dynamics of assetprices. In quantum theory one typically considers bipartite interactions (although in some problems, especially in quantum field theory, more complicated interactions are also explored).

As was already pointed out, the quantum state dynamics in the presence of non-negligible influence of a bath cannot be formulated in terms of pure states represented by normalized vectors in the complex Hilbert space. The characteristics of the finance market demand to introduce an environment based dynamics. One has to reformulate Schrödinger's dynamics in terms of density operators. Such a reformulation leads to von Neumann's equation:

$$
\frac{d \rho}{d t}(t)=-\frac{i}{\gamma}[\mathcal{H}, \rho(t)] .
$$

In the next section we will consider a modification of equation (11) in the presence of a financial environment.

\subsection{Decision making based on Markovian quantum mas- ter equation}

One of the main distinguishing features of the solutions of the Markovian quantum master equation is that, for a wide class of equations, a nonstationary solution $\rho(t)$ stabilizes to a stationary solution $\rho_{\text {prog }}$ representing the long term prognosis (i.e. at some future point in time) of the distribution of the asset-prices.

As was already emphasized, opposite to Schrödinger's equation, the quantum master equation can transform pure states into mixed states. Such a process is called decoherence. This is a dynamical equation in the space of density operators. Therefore the limiting prognosis state $\rho_{\text {prog }}$ can be a mixed state even if the initial price configuration was a pure state. Such a steady state is (under natural conditions) diagonal in the price basis and hence, it represents the resolution of the quantum-like uncertainties present in superposition and entanglement that are present in the initial state $\rho_{0}$.

In general $\rho_{\text {prog }}$ determines only the probabilities for the price values. For example, consider only two assets $\mathcal{A}_{1}, \mathcal{A}_{2}$. If e.g.,

$$
\rho_{\text {prog }}=P\left|n_{1} m_{1}\right\rangle\left\langle n_{1} m_{1}|+Q| n_{2} m_{2}\right\rangle\left\langle n_{2} m_{2}\right|, P+Q=1, P, Q \geq 0,
$$


then the probability that $p_{1}=n_{1}, p_{2}=m_{1}$ equals to $P$ and the probability that $p_{1}=n_{2}, p_{2}=m_{2}$ equals to $Q$; the probabilities of other price configurations equal to zero. We again remark that the prognosis-states, e.g., (12), are in some sense classical states. Superposition indeterminacy, present in the initial state, say $\rho_{0}$ is one of Bell's states (4), disappears in the process of decoherence. This is a most typical scenario of the states' evolution, driven by quantum Markov master equation.

Let us formulate the Markovian approximation of the quantum master equation, the Gorini-Kossakowski-Sudarshan-Lindblad (GKSL) equation [50]:

$$
\frac{d \rho}{d t}(t)=-\frac{i}{\gamma}[\mathcal{H}, \rho(t)]+L(\rho(t)),
$$

where $\mathcal{H}$ is a Hermitian operator acting in $H$ and $L$ is a linear operator acting in the space of linear operators $B(H)$ in $H$ (such maps are often called superoperators). Typically the operator $\mathcal{H}$ represents the state dynamics in the absence of environment, cf. with von Neumann equation (11). However, $\mathcal{H}$ can also contain a contribution of the impact of the environment. The superoperator $L$ has to map density operators into density operators, i.e., it has to preserve "Hermitianity", positive definiteness, the trace. These conditions constraint essentially the class of possible generators $L$. By adding some additional conditions, the so called complete positive definiteness, one can describe the class of generators precisely, see, e.g., [50]. They have the form

$$
L \rho=\sum_{k} \alpha_{k}\left[C_{k} \rho C_{k}^{*}-\left(C_{k}^{*} C_{k} \rho+\rho C_{k}^{*} C_{k}\right) / 2\right]=\sum_{k} \alpha_{k}\left[C_{k} \rho C_{k}^{*}-\frac{1}{2}\left\{C_{k}^{*} C_{k}, \rho\right\}\right] .
$$

Operators $C_{k}$ encode the special features of the social environment.

In the above devised mathematical model the equilibrium state, is determined as $t$ approaches infinity. Of course, in the setting of a financial market the investment decisions have to be taken during a finite time interval. It is natural to assume that a decision maker terminates the process by setting the threshold for state-fluctuations, quantified by some small parameter $\epsilon$. Thus, when the magnitude of the price state fluctuations becomes less than the $\epsilon$, the asset price state is fixed with some probability. 


\section{Numerical simulation, an illustrative ex- ample}

Consider two assets, $\mathcal{A}_{1}, \mathcal{A}_{2}$, with possible prices $n_{1}=1,2$ and $n_{2}=1,2$. Thus each asset has one qubit state space with the basis $|1\rangle,|2\rangle$; the pair of assets has the four dimensional state space $\mathbf{C}^{4}$ with the basis $e_{1}=|11\rangle, e_{2}=$ $|12\rangle, e_{3}=|21\rangle, e_{4}=|22\rangle$.

For the numerical simulation we explore the mathematical model from quantum physics - we refer to it as a three level system of ions, [52]: two ions interacting with each other, the strength of interaction is represented by the parameter $V$. There is given a collection of such systems, prepared in the ground state $e_{1}$, the vacuum state (in physics, it is denoted $|00\rangle$, but we do not want to operate with assets of a zero price). These systems of ions are exposed to the light pump of specially selected frequency to move ions from the ground state to one of the states $e_{2}, e_{3}$. The intensity of the light pump is represented by the parameter $A$. The state $e_{4}$ is the so called dark level, i.e., it is unapproachable from the "light levels" $e_{1}, e_{2}, e_{3}$. This characteristic reduces the dimension of the model's state space from four to three, as such, one can operate with the three dimensional complex Hilbert space $\mathbf{C}^{3}$. In the absence of an interaction with a bath, the population of these levels fluctuates. By playing with the frequency of the radiation, the ions' energy levels and the parameters $V$ and $A$, responsible for the strength of interaction, it is possible to move all the ion systems from the ground state $e_{1}$ to $e_{2}$ or $e_{3}$ and vice versa. Again, by adjusting the parameters, it is possible to realize a transition from $e_{1} \rightarrow e_{2}$ much easier than from $e_{1} \rightarrow e_{3}$. However, these transitions are connected with each other i.e., it is impossible to approach high intensity of $e_{1} \rightarrow e_{2}$ transition without a nontrivial, although weak intensity transition from $e_{1} \rightarrow e_{3}$. By using so called pseudo-spin formalism [52] it can be done with a following Hamiltonian ${ }^{6}$ :

$$
\mathcal{H}=\left(\begin{array}{ccc}
V & A & 0 \\
A & 0 & A \\
0 & A & V
\end{array}\right) .
$$

We emphasize that the quantum physical process under consideration is extremely complex. However, the operational description is reduced to the matrix (15).

\footnotetext{
${ }^{6}$ By proceeding operationally we are not interested in the details of the physical considerations of this formalism. We just use the knowledge, see [52], that this operator generates the aforementioned transitions.
} 
Now we remark that in physics it is impossible to create an isolated system interacting with a pump of the fixed frequency radiation. There is always present a sufficiently strong noise radiation. The presence of this background affects crucially the behavior of these systems of ions. Their states fluctuations are suppressed and the elements of density matrix stabilize to some fixed numbers. In the model under consideration, the off-diagonal elements representing an interference between states $e_{i}$ and $e_{j}, i \neq j$, will vanish (in the limit) and the diagonal ones will approach some fixed probabilities. Again, physicists would apply an operational strategy, not searching to describe explicitly the quantum stochastic process of the bath, but instead to apply a phenomenological (super-)operator $L$ in the GKSL-equation (13). The pseudo-spin formalism is again in use $^{7}$ [52], where the bath is operationally described with the aid of the following operator:

$$
J_{z}=\left(\begin{array}{ccc}
1 & 0 & 0 \\
0 & 0 & 0 \\
0 & 0 & -1
\end{array}\right) .
$$

In physics [11] this is the operator of the spin's projection on the $z$-axis. Using $J_{z}$ we introduce the following operator in the space of density operators in $H=\mathbf{C}^{3}$ :

$$
M \rho=J_{z} \rho-\rho J_{z}=\left[J_{z}, \rho\right] .
$$

The simplest phenomenological quantum (super-)operator representing interaction with the bath can be selected in the form:

$$
L \rho=\left[J_{z}, M \rho\right]=\left[J_{z},\left[J_{z}, \rho\right]\right] .
$$

(It can be shown that it can be also represented in the canonical form (14)) Finally, there is the coupling constant $g>0$ describing the strength of interaction of the systems of ions with the bath. The operator $L$ was constructed in such a way that the solution of the corresponding GKSL-equation

$$
\frac{d \rho}{d t}(t)=-\frac{i}{\gamma}[\mathcal{H}, \rho(t)]-g L(\rho(t))
$$

exhibits stronger decoherence for larger interaction constants $g$.

\footnotetext{
${ }^{7}$ It was found that the spin operators can operationally represent the basic features of the radiation bath. This is a purely formal mathematical modeling approach; in fact the stochastic features of the bath don't correspond to the real spins. Therefore, the terminology "pseudo-spin" is applied. Moreover, the real bath is a physical system of large complexity and with an infinite number of degrees of freedom. The below simple description is a consequence of a well developed approximation technique, leading to the quantum Markov dynamics (13), see [50] for details.
} 
We explore how this model works in finance context, by mapping assets' states onto ions' states. Suppose that an expert preparing a financial prognosis decides to start with a pair of some low-priced shares $\mathcal{A}_{1}, \mathcal{A}_{2}$. We remind that in this section the minimal price $n=1$; so the shares with prices $n_{1}=1, n_{2}=1$ are selected. In the model, the initial state is given by the vector $\left|\psi_{0}\right\rangle=|11\rangle$, or in the density operator of a form by $\rho_{0}=\left|\psi_{0}\right\rangle\left\langle\psi_{0}\right|$ (the financial analogue of the ground state in physics). We assume that the assets under consideration are correlated and the parameter $V$ gives the intensity of correlations. We also assume that at the market there is present an "information pump" towards these assets leading to high intensity transition $e_{1} \rightarrow e_{2}$ and essentially lower intensity transition $e_{1} \rightarrow e_{3}$ (heating of these pair of assets). We also assume that the financial context under consideration is such that these transitions are coupled, i.e., it is impossible to approach high intensity of $e_{1} \rightarrow e_{2}$ transition without nontrivial, although weak intensity transition $e_{1} \rightarrow e_{3}$. The intensity of the information pump towards the pair of assets $\mathcal{A}_{1}, \mathcal{A}_{2}$ is given by the parameter $A$. We ask the reader for understanding: for a moment, our aim is mainly to illustrate our general model by relatively simple numerical examples. We are not yet able to establish the explicit correspondence with financial engineering, e.g., to specify the real financial technique of "financial pumping" into the pair of the correlated assets.

Suppose now that an expert $\mathcal{E}$ works on planning of the investment of $S$ dollars into $\mathcal{A}_{1}, \mathcal{A}_{2}$; he plans to buy $x_{j}$ entities of the $\mathcal{A}_{j}$-assent, $j=1,2$. And $\mathcal{E}$ uses (maybe unconsciously) the aforementioned model to describe the possible dynamics of a pair of correlated assets. In the absence of the environment, based on the expectations of the agents of the financial market, the financial pumping can periodically lift the state $e_{1}=|11\rangle$ to the state $e_{2}=|12\rangle$. By estimating the period of oscillations, $\mathcal{E}$ would be able to make a higher profit by investing money solely in $\mathcal{A}_{2}$ and selling it at the instant of time of approaching the latter state. However, the model is complex enough and it is not easy to find explicitly this exact period in time. Of course, the real financial market has to be modeled by taking into account the interaction with the bath. It seems impossible to solve the equation (19) analytically. We performed simulation for the parameters $V=10$ and $A=V / 15$ for a few values of the coupling constant $g$, see Fig.1. Initially $\rho_{11}(0)=1$. We can see that $\rho_{11}(t) \rightarrow 0.5, t \rightarrow \infty$; we also found that $\rho_{22}(t) \rightarrow 0.4, \rho_{33}(t) \rightarrow$ $0.1, \rho_{i j}(t) \rightarrow 0, i \neq j$. Thus, the expectations of $\mathcal{E}$ about the possible state of the financial market (reduced to a pair of assets in our numerical simulation) are partially transferred from $e_{1}$ to (mainly) $e_{2}$ and (essentially less) to $e_{3} .{ }^{8}$

\footnotetext{
${ }^{8}$ The three-level system of ions that we apply in our model has a unique steady state,
} 


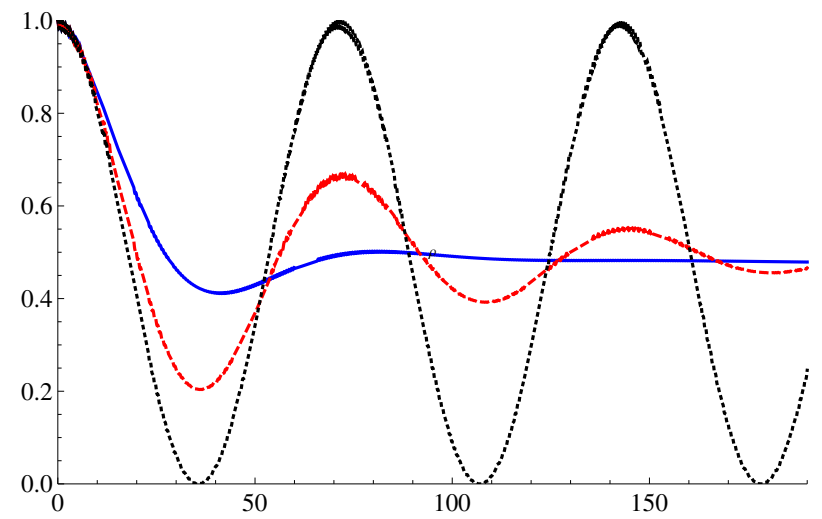

Figure 1: The dynamics of the matrix element $\rho_{11}(t)=\left\langle\rho(t) e_{1} \mid e_{1}\right\rangle$ : Solid line - strong decoherence $(g=A / 30)$; dash line weak decoherence $(g=A / 90)$; dotted line - no decoherence $(g=0)$.

At the equilibrium the state of the prognosis is represented as:

$$
\rho_{\operatorname{prog}}=\left(\begin{array}{ccc}
0.5 & 0 & 0 \\
0 & 0.4 & 0 \\
0 & 0 & 0.1
\end{array}\right) .
$$

We remind that in this equilibrium state the quantum uncertainty has vanished completely. The final stabilized state can be treated as representing the classical probability distribution associated with assets' prices $^{9}$. .

We introduce the portfolio operator (here $x_{1}$ and $x_{2}$ are amounts of money

i.e., by starting with any initial state $\rho_{0}$, its dynamics leads to the same limiting steady state. In particular, if we start with a pure state, which is a superposition of basis vectors with some phases, the final result of observation will not depend on these phases. At the same time, we should note that the dynamics of the system at the initial stage of its evolution depends nontrivially on the phases. In the process of the model application, the expert is only interested in the final state of asset prices that doesn't depend on the initial state and thus doesn't depend on the phases associated with the initial state.

${ }^{9}$ As was pointed out at the very end of the section 3 , an expert in the real financial setting cannot wait infinitely long for the price states to approach a complete stabilization to the equilibrium state. This situation is illustrated in the process of the presented numerical simulation. Here the time-steps are discrete, $t_{1}, t_{2}, \ldots, t_{n}, \ldots$ To stop the calculations, we estimate the difference between the consecutive values of the state-matrix, until some threshold is reached. The matrix (20) presents the probabilities for possible price configurations on the market. As such, the output of this matrix is providing the expert with the probabilistic prognosis of the future price configuration on the finance market. 
which are initially put into $\mathcal{A}_{1}$ and $\mathcal{A}_{2}$, respectively):

$$
A\left(x_{1}, x_{2}\right)=\sum_{i j}\left(i x_{1}+j x_{2}\right)|i j\rangle\langle i j| .
$$

Thus in our special case

$$
A\left(x_{1}, x_{2}\right)=\left(x_{1}+x_{2}\right)|11\rangle\left\langle 11\left|+\left(x_{1}+2 x_{2}\right)\right| 12\right\rangle\left\langle 12\left|+\left(2 x_{1}+x_{2}\right)\right| 21\right\rangle\langle 21| .
$$

We find the average price of this portfolio at the instant of time $t$ as

$$
\left\langle A\left(x_{1}, x_{2}\right)\right\rangle_{\rho(t)} \equiv \operatorname{Tr} \rho(\mathrm{t}) \mathrm{A}\left(\mathrm{x}_{1}, \mathrm{x}_{2}\right) .
$$

And the prognosis for the portfolio price is calculated as

$$
\left\langle A\left(x_{1}, x_{2}\right)\right\rangle_{\rho_{\text {prog }}} \equiv \operatorname{Tr} \rho_{\text {prog }} \mathrm{A}\left(\mathrm{x}_{1}, \mathrm{x}_{2}\right)=0.5\left(\mathrm{x}_{1}+\mathrm{x}_{2}\right)+0.4\left(\mathrm{x}_{1}+2 \mathrm{x}_{2}\right)+0.1\left(2 \mathrm{x}_{1}+\mathrm{x}_{2}\right)
$$

or

$$
\langle A(x, S-x)\rangle_{\rho_{\text {prog }}} \equiv \operatorname{Tr} \rho_{\text {prog }} \mathrm{A}\left(\mathrm{x}_{1}, \mathrm{x}_{2}\right)=0.5 \mathrm{~S}+0.4(2 \mathrm{~S}-\mathrm{x})+0.1(\mathrm{x}+\mathrm{S})=1.4 \mathrm{~S}-0.3 \mathrm{x} .
$$

The profit is calculated as

$$
\langle A(x, S-x)\rangle_{\rho_{\text {prog }}}-S=0.4 S-0.3 x .
$$

Hence, by investing solely to $\mathcal{A}_{2}$ one maximizes the profit approaching the value $0.4 S$.

We remark that, although initially the state of a pair of assets $\mathcal{A}_{1}, \mathcal{A}_{2}$ is a basis state $e_{1}=|11\rangle$, which is characterized by the fixed prices and the output state $\rho_{\text {prog }}$ is the classical mixture of the basis states - the states with the fixed prices, the adaptive dynamics generates the states $\rho(t), 0<t<\infty$, with nonzero interference coefficients, the off-diagonal terms. These states represent deep uncertainty of the nonclassical type about the possible prices. Moreover, one doest need to start ( by mimicking straightforwardly the formalism of physics) from the "ground state" $e_{1}$. It is possible to perform a simulation, beginning with some superposition of the fixed price states, $\psi_{0}=\sum_{i j} c_{i j}|i j\rangle$. We aim proceed with such a development in one of the future works. In such a model, a financial expert $\mathcal{E}$ is not planning for an instantaneous investment, but in some near future. Hence, for $\mathcal{E}$, the initial state would be uncertain as well. Such a model advancement would potentially reflect to a better degree the real process of a financial prognosis for the future investments. 


\section{Concluding remarks}

We remind that classical probability theory (including the Bayesian probability updating scheme and the corresponding modern economic theories of rationality) is fundamentally based on classical Boolean logic. This contribution is concerned with modeling the behavior of decision makers and their subsequent impact on the asset prices. The investors in many contetxt elaborate their investment strategies by using a reasoning based on quantum logic, [51]. The latter essentially relaxes the constraints on the rules of reasoning posed by Boolean logic. ${ }^{10}$

We apply the notion "irrational" in a positive constructive sense, as providing for creative novel solutions, which are impossible in the framework of Boolean logic. In a sense, we can speak of quantum rationality. By using quantum logic, decision makers can proceed with complementary (mutually exclusive) financial contexts and make decisions based on the analysis of such contexts. The Hilbert space formalism provides for the unique possibility to create a unifying mental representation of complementary contexts. ${ }^{11}$ One of the main features of the theory of open quantum systems [50], which is used to model decision making and more broadly a resolution from uncertainty, about the future asset prices is precisely the possibility to describe context-adaptive dynamics, in the very general mathematical setting.

In spite of the aforementioned original features of quantum rationality, it cannot be considered as superior with respect to the classical Boolean (Baeysian) rationality. For example, by getting a novel possibility to unify complementary contexts, a decision maker violates the rules of Boolean reasoning and may generate biases, that are considered as a proof of irrationality from the viewpoint of "a more careful Boolean analysis". The notion of quantum rationality is very close to the notion of bounded rationality, [4]. A decision maker proceeds using the rules of quantum logic and quantum operational update of events' probabilities, because such information processing simplifies the reasoning. It allows the decision maker to take short cuts in her decision making process instead of constructing a complete Boolean model. Such type of reasoning emerged in the process of human evolution; in many

\footnotetext{
${ }^{10}$ As was recently emphasized in the talk of K. Gustafson [38], Boolean logic corresponds to the information processing by computers and artificial intelligence systems, whereas human mind deviates from Boolean reasoning. This claim is based on the results of an extended study of human behavior, [31].

${ }^{11}$ The analysis of various no-go theorems of quantum mechanics, for instance the theorems elaborating against a possibility to reproduce a quantum statistical data on the basis of deterministic models, demonstrates that counter-facts plays an important role in quantum reasoning.
} 
cases it is more important to proceed faster and use less computational resources than to create a (Boolean) rational model of some situation, cf. [5], [12].

As was pointed out in [10], Sir Isaac Newton said that he can calculate the motion of heavenly bodies, but not the behavior of investors. This statement reflects perfectly the main idea behind our study: behavior of investors is intrinsically indeterministic and it does not match with the laws of classical physics, even statistical physics, based on the theory of classical stochastic processes. On the other hand, it might match the methods of quantum physics and might be properly described on the basis of quantum probability, reflecting a deeper type of uncertainty represented by the superposition and entanglement of investors belief-states that lead to deeper uncertainty associated with asset prices.

We hope that this paper and the presented mathematical model of asset price formation, may stimulate the interest to further applications of the mathematical apparatus of quantum theory to the field of behavioral finance and even financial engineering.

\section{References}

[1] H. Takahashi, T. Terano, Agent-Based Approach to Investors' Behavior and Asset Price Fluctuation in Financial Markets. J. Artificial Societies and Social Simulation 6, 3, (2003).

[2] H. Takahashi, H. Takahashi, T Terano, Exploring Risks of Financial Markets through Agent-Based Modeling, SICE-ICASE, 2006. International Joint Conference, 939 - 942, (2006).

[3] J S Bell, Speakable and Unspeakable in Quantum Mechanics Cambridge: Cambridge Univ. Press, (1987).

[4] H. Simon, A behavioral model of rational choice. Quarterly Journal of Economics,69, 99-118, (1955).

[5] H. Simon, Bounded Rationality and Organizational Learning. Organization Science, 2 (1): 125134, (1991).

[6] Ch. P. Kindleberger, Bubbles, The New Palgrave Dictionary of Money and Finance, Vol. 1, pp 243, London: Macmillan (1992)

[7] Ch. P. Kindleberger, Manias, Panics, and Crashes, 3rd Edition, John Wiley \& Sons, Inc. (1996) 
[8] R. Shiller, Irrational Exuberance, Princeton, Princeton University Press, (2000).

[9] J. E. Ingersoll, Theory of Financial Decision Making, Rowman \& Littlefield (1987).

[10] R. E. Bailey, The economics of financial markets. Cambridge, Cambridge University Press, (2005).

[11] G. Jaeger, Quantum Information: An Overview, Springer, HeidelbergBerlin-New York (2007).

[12] G. Gigerenzer, R. Selten, Bounded Rationality: The Adaptive Toolbox. MIT Press.(2002) ISBN 0-262-57164-1.

[13] A. N. Shiryaev, Essentials of Stochastic Finance: Facts, Models, Theory, World Scientific Publ., Singapore, (1999).

[14] R. N. Mantegna, H. E. Stanley, Introduction to Econophysics. Correlations and Complexity in Finance, Cambridge, Cambridge University Press (2007).

[15] B. E. Baaquie, Quantum Finance: Path Integrals and Hamiltonians for Options and Interest Rates. Cambridge, Cambridge University Press, (2004).

[16] B. E. Baaquie. Interest Rates and Coupon Bonds in Quantum Finance. Cambridge University Press, Cambridge, UK, (2009).

[17] O. Choustova, Quantum Bohmian model for financial market, Physica A, 374: 304-314, (2007)

[18] E. Haven, A discussion on embedding the Black Scholes option pricing model in a quantum physics setting, Physica A: Statistical Mechanics and its Applications), 304, 507524 (2002).

[19] E. Haven, Private Information and the "Information Function": A Survey of Possible Uses. Theory and Decision, 64, 193-228 (2008).

[20] E. W. Piotrowski, M. Schroeder, A. Zambrzycka, Quantum extension of European option pricing based on the Ornstein Uhlenbeck process. Physica A: Statistical Mechanics and its Applications, 368, 176-186 (2006).

[21] E. W. Piotrowski, and J. Sladkowski, Quantum solution to the Newcombs paradox, International Journal of Quantum Information, 1, 395402, (2003). 
[22] R. J. Hawkins, B. R. Frieden. Fisher information and equilibrium distributions in econophysics, Physics Letters A, 322, 126-130 (2004).

[23] R. J. Hawkins, M. Aoki, B. R. Frieden. Asymmetric information and macroeconomic dynamics, Physica A, 389: 3565-3571 (2010).

[24] H. Ishio, E. Haven, Information in asset pricing: A wave function approach. Annalen der Physik (Berlin), 18(1), 33-44 (2009).

[25] P. LaMura, Projective Expected Utility. Journal of Mathematical Psychology, 53(5), 408-414, (2009)

[26] M. Schaden, Quantum finance: A quantum approach to stock price fluctuations, Physica A, 316, 511, (2002).

[27] M. Shubik,(1999) Quantum economics, uncertainty and the optimal grid size, Economics Letters, 64 (3): 277-278, (1999).

[28] C. Schinckus, Economic uncertainty and econophysics, PhysicaA, 388, 4415-4423, (2009).

[29] M. Asano, M. Ohya, Y. Tanaka, A. Khrennikov, I. Basieva, On application of Gorini-Kossakowski-Sudarshan-Lindblad equation in cognitive psychology. Open Systems and Information Dynamics, 18,55-69 (2011).

[30] M. Asano, I. Basieva, A. Khrennikov, M. Ohya, Y. Tanaka, Quantumlike generalization of the Bayesian updating scheme for objective and subjective mental uncertainties. Journal of Mathematical Psychology, 56, N 3, 166-175 (2012).

[31] J. Bernasconi, K. Gustafson, Contextual quick learning and generalization by human and machine. Network: Computation in Neural Systems, 9, 85-106 (1994).

[32] J. R. Busemeyer and P. D. Bruza, Quantum models of cognition and decision. Cambridge Press, (2012).

[33] J. R. Busemeyer, Z. Wang, and J. T. Townsend, Quantum dynamics of human decision making. J. Math. Psychology, 50, 220-241 (2006).

[34] J. R. Busemeyer, Z. Wang, A. Lambert-Mogiliansky. Empirical comparison of Markov and quantum models of decision making. J. Math Psychology, 53 (5), 423-433 (2009). 
[35] J. R. Busemeyer,, E. M. Pothos, R. Franco, J. Trueblood, A quantum theoretical explanation for probability judgment errors, Psychological Review, 118, 193-218 (2011).

[36] E.N. Dzhafarov, J.V. Quantum entanglement and the issue of selective influences in psychology: An overview. Lecture Notes in Computer Science, 7620, 184-195 (2012).

[37] E.N. Dzhafarov, J.V. Kujala, All-possible-couplings approach to measuring probabilistic context. PLoS ONE, 8(5):e61712, (2013).

[38] K. Gustafson, The importance of imagination (of lack thereof) in artificial, human, quantum cognition and decision making. Conference paper: Conference on "Quantum Probability and the Mathematical Modeling of decision-making", March 2015, Toronto, Fields Institute. (2015).

[39] E. Haven, A. Khrennikov, Quantum mechanics and violation of the surething principle: the use of probability interference and other concepts, J. Math. Psychology, 53, 378-388 (2009).

[40] E. Haven, A. Khrennikov, Quantum Social Science, Cambridge Press (2012).

[41] P. Khrennikova, Evolution of quantum-like modeling in decision making processes, AIP Conf. Proc. 1508, 108 (2012).

[42] P. Khrennikova, A Quantum Framework for 'Sour grapes' in Cognitive dissonance. Proceedings of Quantum Interaction-13, Lecture Notes in Computer Science, University of Leicester Press (2013).

[43] E. Pothos, J.R. Busemeyer, A quantum probability explanation for violation of rational decision theory. Proc. Royal. Soc. B, 276, 2171-2178, (2009).

[44] W. H. Zurek, Decoherence, einselection, and the quantum origins of the classical. Reviews Of Modern Physics, 75 (7), 715-775, (2003).

[45] E. F Fama, Efficient Capital Markets, A Review of Theory and Empirical Work, Journal of Finance, 25, 383-417 (1970).

[46] E. F Fama, K.R. French, K. R., Common risk factors in the returns on stocks and bonds, Journal of Financial Economics 33 (3) (1993) 
[47] E. F. Fama, K. R. French, A Five-Factor Asset Pricing Model (September 2014), Fama-Miller Working Paper. Available at SSRN: http://ssrn.com/abstract=2287202, ( 2014).

[48] H. Shefrin, M. Statman, Behavioural Capital Asset Pricing Theory, Journal of Financial and Quantitative Analysis, 29(3), 323-249, ( 1994).

[49] H. Baker, N.B. Nofsinger, R. Kolb, Behavioural Finance: Investors, Corporation and Markets, John WileySons (2010).

[50] M. Ohya, I. Volovich, Mathematical foundations of quantum information and computation and its applications to nano- and bio-systems. Springer, Heidelberg-Berlin-New (2011).

[51] J. Birkhoff, J. von Neumann, The logic of quantum mechanics. Annals of Mathematics, 37, N 4, 823-843 (1936).

[52] R. H. Dicke, Coherence in Spontaneous Radiation Processes. Phys. Rev, 93, 99 (1954). 